\title{
Reweighting sequential effects across different distributions of foreperiods: Segregating elementary contributions to nonspecific preparation
}

\author{
SANDER A. LOS and FRANK AGTER \\ Vrije Universiteit, Amsterdam, The Netherlands
}

\begin{abstract}
In the study of nonspecific preparation, the response time (RT) to an imperative stimulus is analyzed as a function of the foreperiod (FP), the interval between a warning stimulus and the imperative stimulus. When FP is varied within blocks of trials, a downward sloping FP-RT function is usually observed. The slope of this function depends on the distribution of FPs (the more negative the skewness, the steeper the slope) and on intertrial sequences of FP (the longer the FP on the preceding trial, the steeper the slope). Because these determinants are confounded, we examined whether FP-RT functions, observed under three different FP distributions (i.e., uniform, exponential, and peaked) can be predicted, one from the other, by reweighting sequential effects. It turned out that reweighting explained very little variance of the difference between the FP-RT functions, suggesting a dominant role of temporal orienting strategies.
\end{abstract}

A general goal of cognitive science is to uncover elementary processes that contribute to observed behavior (e.g., Sternberg, 2001). Once these processes are uncovered, scientists may start to describe them individually and show how they operate in mutual interaction.

This goal has recently become topical in the study of nonspecific preparation. Nonspecific preparation is concerned with the process of attaining and maintaining general preparedness for action to an anticipated imperative stimulus (e.g., Gottsdanker, 1975; Niemi \& Näätänen, 1981). This process is nonspecific in the sense that it is not concerned with a particular stimulus, response, or stimulus-response mapping, but merely with the timing of the imperative stimulus. Thus, nonspecific preparation has been studied by varying the foreperiod (FP), the interval between the offset of a neutral warning stimulus $\left(\mathrm{S}_{1}\right)$ and the onset of the imperative stimulus $\left(\mathrm{S}_{2}\right)$. Participants are instructed to respond as quickly as possible to $\mathrm{S}_{2}$ according to some task rule. On the assumption that the response time (RT) is shorter as the state of nonspecific preparation is higher, the participant's state of nonspecific preparation at the moment of $\mathrm{S}_{2}$ presentation can be inferred (see Niemi \& Näätänen, 1981, for a review).

Below we will argue that, in spite of its apparent simplicity, nonspecific preparation is not a unitary process and encompasses at least two more elementary processes.

The authors contributed equally to this study. Correspondence should be addressed to S. A. Los, Department of Cognitive Psychology, Vrije Universiteit, Van der Boechorststraat 1, 1081 BT Amsterdam, The Netherlands (e-mail: sa.los@psy.vu.nl).
Although these processes are not necessarily mutually exclusive, they may be competitors in accounting for specific phenomena. In the present article, we will address one such a phenomenon: the effect of the distribution of FP across the trials of a block. To specify the problem, we will first present some basic findings obtained in the variable-foreperiod design, which provides the basis for inferences about distinct processes. Then, we will show that the relative contribution of these processes cannot be determined unambiguously in studies that varied the distribution of FP. We present a method that is aimed at disentangling these contributions, and show its application to new experimental data.

\section{The Variable-Foreperiod Design}

In the variable-foreperiod design, FP is varied randomly across the trials of a block. As a result, $\mathrm{S}_{2}$ may appear at one of several possible moments relative to the offset of $\mathrm{S}_{1}$. These moments are here referred to as critical moments. The moment at which $\mathrm{S}_{2}$ is presented on any particular trial is referred to as the imperative moment of that trial. When the distribution of FP is uniform - that is, when each critical moment has an equal chance of becoming the imperative moment on each trial-mean RT has been shown to be a (negatively accelerated) downward sloping function of FP (e.g., Drazin, 1961; Los \& Van den Heuvel, 2001; Woodrow, 1914; Zahn, Rosenthal, \& Shakow, 1963).

One influential account of this finding has been that nonspecific preparation develops in accordance with the conditional probability of $\mathrm{S}_{2}$ occurrence (e.g., Elithorn \& Lawrence, 1955; Jurkowski, Stepp, \& Hackley, 2005; 
Luce, 1986; Näätänen, 1970; Sperling \& Dosher, 1986). Under a uniform FP distribution, this conditional probability is low after the offset of $\mathrm{S}_{1}$, increases as critical moments are bypassed without occurrence of $\mathrm{S}_{2}$, to reach unity prior to the last critical moment. Consistent with this proposal, it has been shown that the FP-RT function becomes about flat when an exponential FP distribution is used, such that the conditional probability of $\mathrm{S}_{2}$ occurrence during FP is equal for each critical moment (e.g., Näätänen, 1971; Trillenberg, Verleger, Wascher, Wauschkuhn, \& Wessel, 2000).

In spite of its parsimony, this proposal has a major shortcoming, however. It fails to recognize that the FPRT function has limited significance in itself because it derives its shape, at least to a large extent, from an asymmetric sequential effect of FP. This effect implies that RT on any trial $n$ is longer when the FP on trial $n\left(\mathrm{FP}_{n}\right)$ was preceded by a longer FP on trial $n-1\left(\mathrm{FP}_{n-1}\right)$ than when it was preceded by an equally long or shorter $\mathrm{FP}_{n-1}$ (e.g., Baumeister \& Joubert, 1969; Karlin, 1959; Los, Knol, \& Boers, 2001; Woodrow, 1914; Zahn et al., 1963). In particular, it has been shown that the FP-RT function is about flat after the shortest $\mathrm{FP}_{n-1}$ (e.g., Los et al., 2001; Van der Lubbe, Los, Jaśkowski, \& Verleger, 2004; Zahn et al., 1963) and becomes increasingly steeper as $\mathrm{FP}_{n-1}$ increases. That is, the negative slope of the average FP-RT function is almost entirely determined by the asymmetric sequential effect, and since the conditional probability of $\mathrm{S}_{2}$ occurrence does not account for this effect, it should be discarded as a general account of FP effects.

In view of this, a crucial question in the variableforeperiod design is: What causes the sequential effect of FP? According to the trace conditioning account, first proposed by Los (1996; see also Los \& Heslenfeld, 2005; Los et al., 2001; Los \& Van den Heuvel, 2001), each critical moment has a conditioned strength associated with it. This conditioned strength can be conceived of as a memory trace that determines the level of nonspecific preparation to be attained when its associated critical moment is reached during FP. The state of conditioning is adjusted on a trial-by-trial basis in accordance with three learning rules. First, as a critical moment is bypassed during FP, its associated conditioned strength is decreased (i.e., subject to extinction). Second, when the response to $\mathrm{S}_{2}$ is released, the conditioned strength associated with the imperative moment is increased (i.e., reinforced). Third, for critical moments beyond the imperative moment, the associated conditioned strength is left unchanged. This explains the asymmetry of sequential effects: Conditioned strengths associated with critical moments prior to the imperative moment are subject to extinction, whereas conditioned strengths associated with later critical moments are not. Los et al. (2001) showed that a formalized version of this model, as adapted from the literature on animal timing (Machado, 1997), provides a good quantitative fit to an extensive data set of sequential effects. ${ }^{1}$

According to the trace conditioning account, the sequential effect of FP is an unintentional consequence of extinction and reinforcement processes. As such, the nature of the processing underlying the sequential effect is distinct from what Nobre and colleagues have called temporal orienting (e.g., Nobre, 2001). Temporal orienting is an intentional process that involves the focusing of attentional resources to a future point in time at which a significant event is expected. This ability has been demonstrated in cuing studies, in which $\mathrm{S}_{1}$ is a symbolic cue that provides either no information (neutral cue), valid information, or invalid information about the duration of the impending FP. Valid cues are typically presented much more frequently than invalid cues to encourage participants to use the information provided by the cue. Cuing studies have shown that responding at early critical moments is faster for valid cues than for neutral cues and is faster for neutral cues than for invalid cues. By contrast, responding at late critical moments is not affected by the information value of the cue (e.g., Coull, Frith, Büchel, \& Nobre, 2000; Coull \& Nobre, 1998; Kingstone, 1992, Experiment 4; Los \& Heslenfeld, 2005; Los \& Van den Heuvel, 2001; Miniussi, Wilding, Coull, \& Nobre, 1999; Zahn, 1970). These findings are consistent with the view that temporal orienting is initially directed to the moment specified by the cue and may be redirected if $\mathrm{S}_{2}$ turns out to appear later than when specified, but not vice versa (e.g., Alegria, 1975; Coull \& Nobre, 1998).

By dissociating sequential effects of FP from effects of cuing, Los and colleagues provided evidence that temporal orienting and trace conditioning are distinct processes. In particular, in a cuing study, Los and Van den Heuvel (2001) observed sequential effects that were as strong in the invalid-cue condition as in the neutral-cue condition. That is, sequential effects were unimpaired when the invalid cue had drawn the focus of temporal orienting away from the impending imperative moment. Furthermore, Los and Heslenfeld (2005) showed that $\mathrm{FP}_{n-1}$ and cuing (neutral or valid) had largely independent effects on the contingent negative variation, an event-related brain potential reflecting nonspecific preparation during FP. Both findings suggest that the sequential effect of FP is caused by an unintentional process, in agreement with the trace conditioning account.

To summarize, studies using the variable-foreperiod design have yielded evidence for two distinct contributions to the state of nonspecific preparation. Temporal orienting is an intentional contribution, which has been demonstrated in cuing studies. Trace conditioning is an unintentional contribution, which accounts for sequential effects of FP. Thus, to the extent the shape of the FP-RT function is determined by sequential effects (which appears to be strongly the case under a uniform FP distribution), it reflects unintentional processes of trace conditioning.

\section{Effects of Foreperiod Distribution}

An important determinant of the slope of the average FP-RT function is the FP distribution. In particular, it has been shown that the FP-RT function becomes flatter to the extent that the FP distribution becomes more positively 
skewed (i.e., by increasing the probability that $S_{2}$ occurs at relatively early critical moments; e.g., Baumeister \& Joubert, 1969; Näätänen, 1971; Trillenberg et al., 2000; Zahn \& Rosenthal, 1966) and steeper to the extent that the FP distribution becomes more negatively skewed (i.e., by increasing the probability that $\mathrm{S}_{2}$ occurs at relatively late critical moments; e.g., Baumeister \& Joubert, 1969; Zahn \& Rosenthal, 1966).

Extending the discussion of the previous section, a relevant question now is whether the effect of FP distribution also reflects the asymmetry of sequential effects, and, by inference, the operation of trace conditioning processes. To see that this might be the case, consider the consequences of making the skewness of an FP distribution increasingly more positive starting from a negatively skewed distribution. Increasing the positivity of the skewness implies that early critical moments are increasingly less frequently bypassed during a block of trials. As a result, mean RT at early critical moments is increasingly less impaired by long FPs on preceding trials, which might explain the flattening of the FP-RT function.

However, it could be that the differential involvement of specific intertrial transitions plays a relatively minor role in accounting for effects of FP distribution. It is possible that the distribution of FP guides the participant's strategy of temporal orienting (e.g., Milliken, Lupiáñez, Roberts, \& Stevanovski, 2003; Trillenberg et al., 2000). This is a viable possibility, because cuing studies have shown that valid preknowledge about the duration of the impending FP helps participants compensate for the negative influences of preceding long FPs (Los \& Heslenfeld, 2005; Los $\&$ Van den Heuvel, 2001). This suggests the possibility that participants use the properties of a nonuniform FP distribution to achieve a similar compensation. In particular, if participants treat the a priori most probable moment of $\mathrm{S}_{2}$ occurrence as an implicit cue, temporal orienting to that moment may well overrule the contribution of trace conditioning.

\section{Reweighting Sequential Effects}

Building on an old suggestion by Drazin (1961; see also Baumeister \& Joubert, 1969; Zahn \& Rosenthal, 1966), we developed and applied a method that enables a precise estimate of the contribution of sequential effects to the difference between FP-RT functions observed under different FP distributions. To this end, we used three FPs of 300,600 , and $1,200 \mathrm{msec}$, and we selected three distributions on the basis of high mutual distinctiveness: uniform, exponential, and peaked (see also Trillenberg et al., 2000). The exponential distribution was "nonaging," in that the conditional probability of $S_{2}$ occurrence at each critical moment was equal during the FP. In the peaked distribution, there was an increased probability for $\mathrm{S}_{2}$ to occur at the central critical moment.

To exclude differential sequential effects as a source of variance between the different FP distributions, we put those distributions on par with regard to the frequency of the various intertrial transitions. Specifically, we re- weighted the mean RT for each of the specific intertrial transitions occurring under distribution $g$ with the frequency of those transitions occurring under distribution f. Formally,

$$
\widehat{\mathrm{RT}}_{i}(f \leftarrow g)=\sum_{j} \frac{\mathrm{RT}_{i j}(g) \times N_{i j}(f)}{N_{i}(f)},
$$

where $i$ and $j$ denote the FP on trials $n$ and $n-1$, respectively, $f$ and $g$ denote two different FP distributions, and $N$ is the number of observations. The expression on the left side of Equation 1 denotes an estimated RT of foreperiod $i$ under distribution $f$ as predicted by distribution $g$. This prediction, specified on the right side of Equation 1, implies a reweighting of the specific intertrial transitions of FP occurring under distribution $g$ by their proportion of occurrence under distribution $f$.

By calculating $\widehat{\mathrm{RT}}_{i}$ for each FP in the design, an FP-RT function for distribution $f$ is obtained that is predicted from distribution $g$. If, on the one hand, this reweighting procedure perfectly predicts the FP-RT function under distribution $f$ [i.e., $\widehat{\mathrm{RT}}(f \leftarrow g)=\mathrm{RT}(f)]$, the observed difference between the FP-RT functions under distributions $f$ and $g$ is exclusively caused by the unequal frequency of the different intertrial transitions. If, on the other hand, the observed difference between the FP-RT functions under distributions $f$ and $g$ is not reduced after reweighting [i.e., $\widehat{\mathrm{RT}}(f \leftarrow$ $g)=$ RT $(g)]$, sequential effects are irrelevant to the observed difference. In general, the proportion of the difference between the FP-RT functions under two different FP distributions that is accounted for by sequential effects is given by

$$
1-\frac{\sum_{i} \mathrm{RT}_{i}(f)-\widehat{\mathrm{RT}}_{i}(f \leftarrow g)}{\sum_{i} \mathrm{RT}_{i}(f)-\mathrm{RT}_{i}(g)},
$$

where, as before, $i$ indexes FP on trial $n$, and $f$ and $g$ are different FP distributions. To the extent Equation 2 yields outcomes closer to 1, the different FP-RT functions for different distributions are attributable to sequential effects and thus reflect processes of trace conditioning. To the extent Equation 2 yields outcomes closer to 0, a role of another process, possibly temporal orienting, is indicated, in addition to or overruling the contribution of trace conditioning.

\section{METHOD}

\section{Participants}

Nine undergraduate students of the Vrije Universiteit (8 women and 1 man; age range $=19-22$ years) took part in three 2 -h sessions in exchange for $\mathrm{a} € 45$ fee. All participants had normal or correctedto-normal vision and did not know the purpose of the experiment.

\section{Materials and Stimuli}

The laboratory consisted of six identical dimly lit, air-conditioned cubicles. Each cubicle was equipped with a personal computer connected to a 17-in. monitor and a standard QWERTY keyboard. The software package ERTS (Beringer, 1992) was used for the layout and 
timing of the experimental trials. The participants sat approximately $90 \mathrm{~cm}$ in front of the monitor, with their index fingers resting on the shift keys, which were used as the response buttons. $\mathrm{S}_{1}$ was a "+" sign, consisting of two $0.83^{\circ} \times 0.19^{\circ}$ crossed bars, and $\mathrm{S}_{2}$ was a $0.83^{\circ} \times 0.83^{\circ}$ square. All stimuli were displayed in white on a black background.

\section{Procedure}

Every trial started with the presentation of $S_{1}$ on the middle of the screen, which the participants were instructed to fixate. After $200 \mathrm{msec}$, the horizontal bar of $\mathrm{S}_{1}$ was erased, indicating the start of FP. FP lasted 300,600 , or $1,200 \mathrm{msec}$, after which $\mathrm{S}_{2}$ appeared equiprobably to the right or the left of the vertical bar at a visual angle of $2.4^{\circ}$ (center to center). The participants were instructed to make a spatially compatible response to $S_{2}$ by pressing the right or the left shift key as fast as possible. The vertical bar and $\mathrm{S}_{2}$ remained on the screen until a response was given or after a fixed interval of $600 \mathrm{msec}$ had elapsed since the onset of $\mathrm{S}_{2}$, whichever occurred earlier. On catch trials, no $\mathrm{S}_{2}$ was presented, and the participants were instructed not to respond. On these trials, the screen turned blank after an interval of $1,500 \mathrm{msec}$. A $50-\mathrm{msec}, 50-\mathrm{Hz}$ tone was used to indicate an error. Consecutive trials were separated by a blank screen for $1,000 \mathrm{msec}$.

The independent variables were FP distribution (uniform, exponential, or peaked) and FP $(300,600$, or $1,200 \mathrm{msec})$. Both were varied within subjects. In the uniform condition, the ratio of occurrence of trials with an FP of 300,600 , or 1,200 msec or catch trials was $1: 1: 1: 1$. In the exponential condition, the ratio was $4: 2: 1: 1$; and in the peaked condition, the ratio was 1:5:1:1. The FPs were randomly distributed across trials given the constraints of the FP distributions. The three FP distributions were presented in separate sessions on separate days, and their order of presentation was counterbalanced across participants.

Every participant completed the three sessions within a period of 3-5 days. In each of these sessions, they completed 36 blocks of 64 trials each for a single FP distribution. The first block of each session served as practice. Prior to this block, the participants were instructed to respond as fast as possible while maintaining high accuracy. They were also informed of the a priori probabilities of the FPs that applied during that session. After every block, the participants were informed on the screen of their mean RT and their number of errors. Between blocks, the participants were allowed a short break.

\section{Data Analysis}

The data of the practice blocks and the first two trials of each subsequent block were discarded. Also discarded were trials on which the RT was under $150 \mathrm{msec}$ or over $600 \mathrm{msec}(0.44 \%)$ and trials following trials on which the participants responded erroneously. In a first analysis, the mean RTs, computed over correct trials, and percentage of errors were subjected to separate univariate ANOVAs with repeated measures. The Huynh-Feldt correction was applied in all tests involving variables with more than two levels to correct for possible violations of the sphericity of the variance-covariance matrix (e.g., Stevens, 1992). An alpha level of .05 was used for all statistical tests. In a second analysis, we applied Equation 1 to examine the contribution of sequential effects to the difference between the FP-RT functions under the three distributions.

\section{RESULTS}

\section{Observations}

Figure 1 shows mean RTs and mean error percentages as a function of $\mathrm{FP}$ distribution, $\mathrm{FP}_{n}$, and $\mathrm{FP}_{n-1}$. Table 1 summarizes the results of the ANOVA. The percentage of errors remained below $3 \%$ in all conditions. The percentage of errors increased by lengthening $\mathrm{FP}_{n}$ and decreased by lengthening $\mathrm{FP}_{n-1}$.

Regarding the RT data, the significant three-way interaction among $\mathrm{FP}$ distribution, $\mathrm{FP}_{n-1}$, and $\mathrm{FP}_{n}$ prompted us to analyze the simple (interaction) effects for the different $\mathrm{FP}_{n} \mathrm{~s}$. The effect of $\mathrm{FP}_{n-1}$ was significant for the $\mathrm{FP}_{n}$ of $300 \mathrm{msec}\left[F(3,24)=22.70, M S_{\mathrm{e}}=76.70, p<.01\right]$,

$\mathrm{FP}_{n}: 300 \mathrm{msec}$

$\mathrm{FP}_{n}: 600 \mathrm{msec}$

$\mathrm{FP}_{n}: 1,200 \mathrm{msec}$

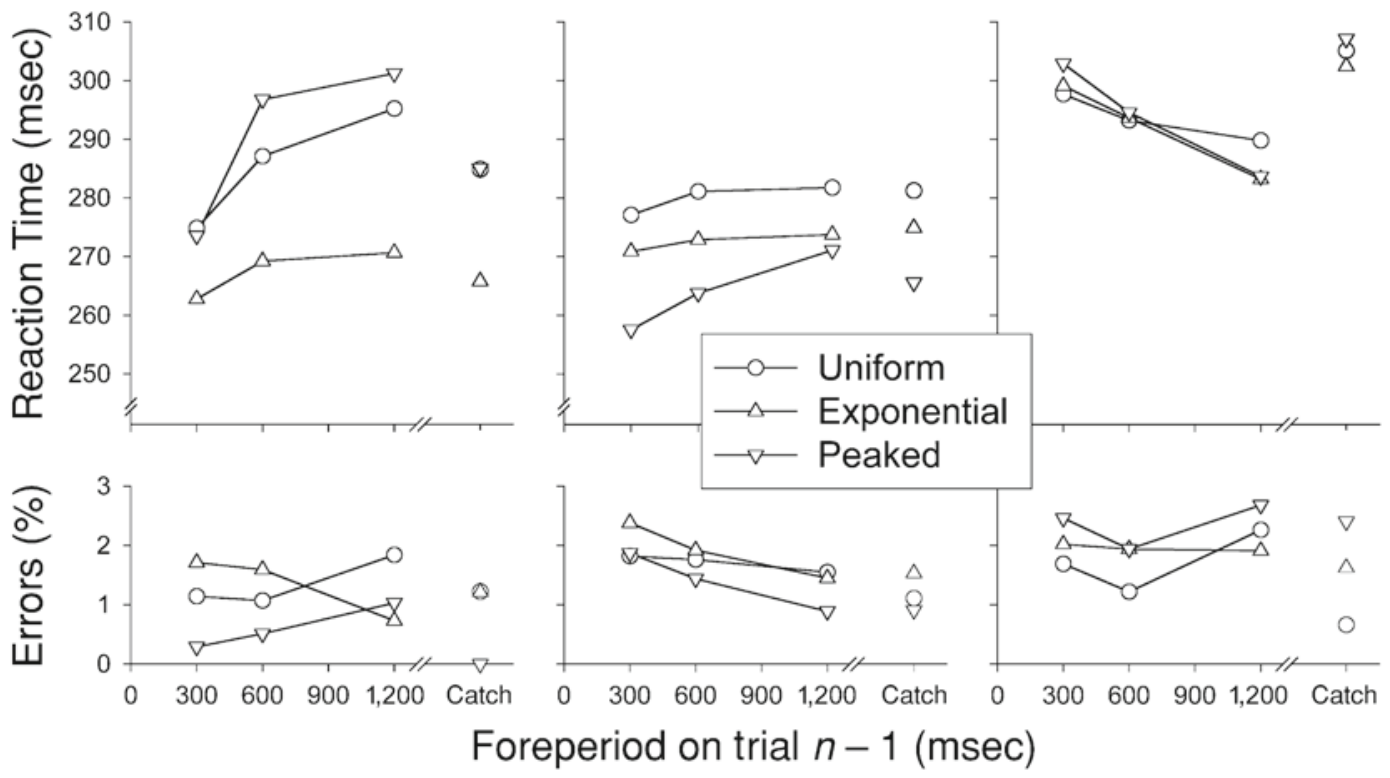

Figure 1. Mean reaction time and mean percentage of errors as a function of the foreperiod on trial $n\left(\mathrm{FP}_{n}\right)$, the foreperiod of trial $n-1$, and the distribution of foreperiods. 
Table 1

Summary of the ANOVA of Reaction Times (RTs) and the Percentage of Errors (PE)

\begin{tabular}{|c|c|c|c|c|c|}
\hline \multirow[b]{2}{*}{ Source } & \multirow[b]{2}{*}{$d f$} & \multicolumn{2}{|c|}{ RT } & \multicolumn{2}{|c|}{$\mathrm{PE}$} \\
\hline & & $F$ & $M S_{\mathrm{e}}$ & $F$ & $M S_{\mathrm{e}}$ \\
\hline Distribution (D) & 2,16 & 0.73 & $3,118.69$ & 0.67 & 4.93 \\
\hline Foreperiod on trial $n\left(\mathrm{FP}_{n}\right)$ & 2,16 & $24.04^{* *}$ & 667.51 & $9.29^{* *}$ & 3.01 \\
\hline Foreperiod on trial $n-1\left(\mathrm{FP}_{n-1}\right)$ & 3,24 & $4.99^{*}$ & 225.69 & $3.09^{*}$ & 1.32 \\
\hline $\mathrm{D} \times \mathrm{HP}_{n}$ & 4,32 & $15.76^{* *}$ & 160.53 & 3.50 & 4.37 \\
\hline $\mathrm{D} \times \mathrm{FP}_{n-1}$ & 6,48 & $2.45^{*}$ & 46.52 & 1.42 & 1.22 \\
\hline $\mathrm{FP}_{n} \times \mathrm{FP}_{n-1}$ & 6,48 & $21.09^{* *}$ & 92.83 & 0.88 & 3.90 \\
\hline $\mathrm{D} \times \mathrm{FP}_{n} \times \mathrm{FP}_{n-1}$ & 12,96 & $2.69^{* *}$ & 39.62 & 0.45 & 3.58 \\
\hline
\end{tabular}

for the $\mathrm{FP}_{n}$ of $600 \mathrm{msec}\left[F(3,24)=6.72, M S_{\mathrm{e}}=36.25\right.$, $p<.01]$, and for the $\mathrm{FP}_{n}$ of $1,200 \mathrm{msec}[F(3,24)=12.37$, $\left.M S_{\mathrm{e}}=151.34, p<.01\right]$. For the $\mathrm{FP}_{n}$ of $300 \mathrm{msec}$, there was also a significant interaction between $\mathrm{FP}_{n-1}$ and $\mathrm{FP}$ distribution $\left[F(6,48)=4.67, M S_{\mathrm{e}}=42.25, p<.01\right]$. As is clear from Figure 1, left panel, this interaction indicates that sequential effects for the $\mathrm{FP}_{n}$ of $300 \mathrm{msec}$ were strongest under the peaked distribution and were the least strong, though still significant $\left[F(3,24)=3.99, M S_{\mathrm{e}}=\right.$ $45.89, p<.05]$ under the exponential distribution. For the $\mathrm{FP}_{n}$ of $600 \mathrm{msec}$, the effect of distribution approached significance $\left[F(2,16)=2.85, M S_{\mathrm{e}}=790.76, p=.087\right]$.

For all distributions, RTs were longer when $\mathrm{FP}_{n-1}$ was longer than $\mathrm{FP}_{n}$ than when it was as long as or shorter than $\mathrm{FP}_{n}$. This effect was small but still significant for the FP of $600 \mathrm{msec}$, where RT was longer after either a catch trial or an $\mathrm{FP}_{n-1}$ of $1,200 \mathrm{msec}(275 \mathrm{msec})$ than after an $\mathrm{FP}_{n-1}$ of either 300 or $600 \mathrm{msec}(270 \mathrm{msec})\left[F(1,8)=8.14, M S_{\mathrm{e}}=\right.$ $9.95, p<.05]$. An exception to the rule that RT increases by lengthening $\mathrm{FP}_{n-1}$ is observed for the condition in which an $\mathrm{FP}_{n}$ of 300 msec occurs after a catch trial, which can be conceived of as a trial with the longest FP. In this condition, RT was shorter $(278 \mathrm{msec})$ than after an $\mathrm{FP}_{n-1}$ of either 600 or $1,200 \mathrm{msec}(286 \mathrm{msec})[F(1,8)=24.05$, $\left.M S_{\mathrm{e}}=12.25, p<.01\right]$.

The FP-RT function under each distribution featured an unusual upward slope toward the longest $\mathrm{FP}_{n}$ of $1,200 \mathrm{msec}$. We examined higher order sequential effects for this $\mathrm{FP}_{n}$, to see whether this finding derives from the strong sequential effects of catch trials. We limited this analysis to the uniform distribution, where we had sufficient observations for the relevant intertrial transitions. When preceded by an increasing number of catch trials, RT for the $\mathrm{FP}_{n}$ of 1,200 msec increased from $302 \mathrm{msec}$ (when trial $n-1$ was a catch trial, but trials $n-2$ and $n-3$ were not) via $315 \mathrm{msec}$ (when both trials $n-1$ and $n-2$ were catch trials but trial $n-3$ was not) to $317 \mathrm{msec}$ (when the three preceding trials were all catch trials). Paired $t$ tests revealed that the second and the third value differed significantly from the first value $[t(8)=$ $4.61, p<.01$, and $t(8)=2.81, p<.05$, respectively, but not from each other, $t(8)=0.36, p=.73]$. When preceded by an increasing number of FPs of 1,200 msec, the RT for the $\mathrm{FP}_{n}$ of $1,200 \mathrm{msec}$ was $290 \mathrm{msec}$ when $\mathrm{FP}_{n-1}$ was 1,200 msec and $\mathrm{FP}_{n-3}$ had a different level (the level of $\mathrm{FP}_{n-2}$ failed to make a difference here) to $279 \mathrm{msec}$ (when $\mathrm{FP}_{n-1}, \mathrm{FP}_{n-2}$, and $\mathrm{FP}_{n-3}$ were all 1,200 msec). Paired $t$ tests indicated that these RTs differed significantly $[t(8)=2.66, p<.05]$.

\section{Reweighting Sequential Effects}

The observed mean FP-RT functions stemming from each combination of two FP distributions are presented in Figure 2, along with the reweighted FP-RT functions that derive from applying Equation 1. Table 2 shows the percentage of the difference between each pair of observed FP-RT functions that is accounted for by reweighting sequential effects, as derives from Equation 2. Two general results should be noted. First, the gain obtained by reweighting was generally small, indicating that sequential effects contributed little to the difference between the FP-RT functions. There was a modest gain of reweighting sequential effects under the peaked distribution to predict the FP-RT functions of the other two distributions and of reweighting sequential effects under the uniform distribution to predict the FP-RT functions under the exponential distribution. Nothing was gained from reweighting sequential effects under the exponential distribution, and the FP-RT function under the peaked distribution could not be predicted from any of the other distributions.

Second, the gain obtained by reweighting was not symmetrical for different distributions. In particular, some gain was obtained by reweighting sequential effects under the uniform or peaked distribution to predict the FP-RT function under the exponential distribution, whereas no gain was obtained by reweighting in the opposite direction. The reason for this asymmetry becomes clear when it is realized that, if any gain is obtained at all, it is mainly due to reducing the distance between FP-RT functions for the shortest FP (see Figure 2). In turn, as shown in Figure 1, under the exponential distribution, sequential effects for the shortest FP are very small, such that almost nothing is achieved by reweighting them. Under the other two distributions, the sequential effects for the shortest FP are larger, which explains the modest gain of reweighting.

In the uniform distribution, there were sufficient observations per condition to justify reweighting up until 


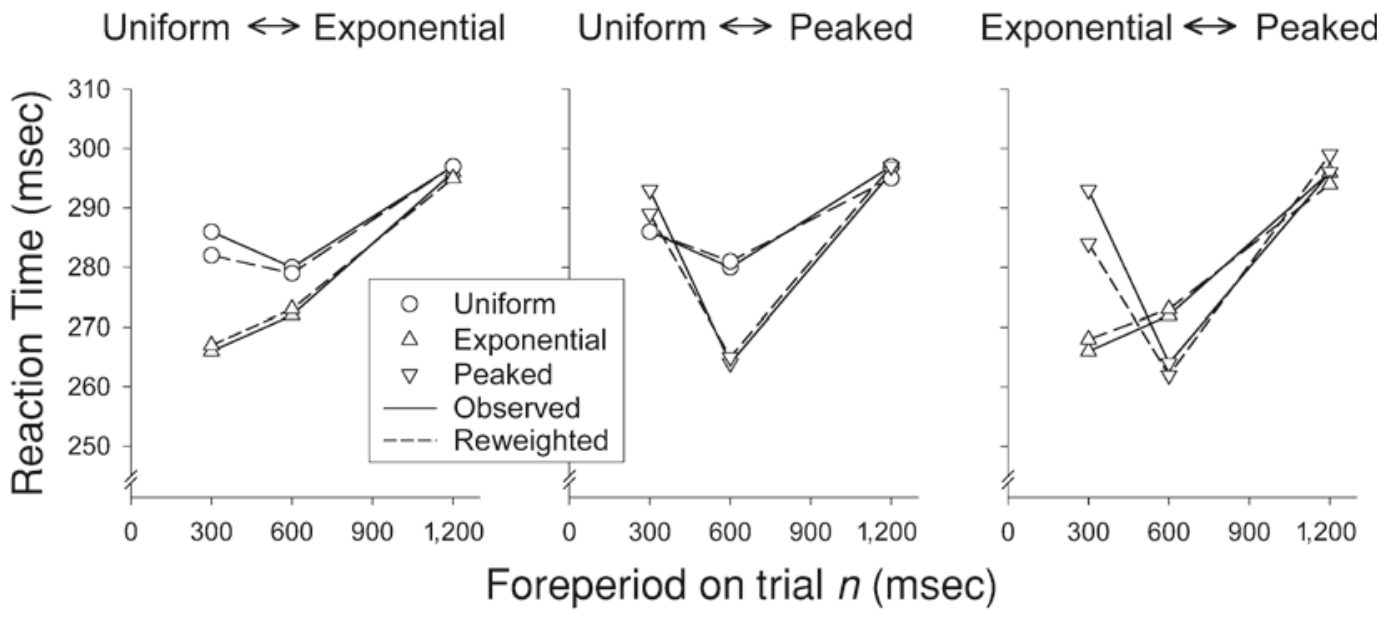

Figure 2. Observed and reweighted mean reaction time as a function of the foreperiod on trial $\boldsymbol{n}$ and the distribution of foreperiods. In each panel, sequential effects as observed under one distribution are reweighted according to their frequency of occurrence under the other distribution using Equation 1.

the second-order sequential effect. ${ }^{2}$ The second-order reweighting explained $27.27 \%$ of the difference between the uniform and the exponential distribution, which exceeds the first-order prediction by $10.18 \%$. Second-order reweighting again completely failed to account for the difference between the uniform and the peaked distribution, yielding a gain of $-0.39 \%$. In the exponential and the peaked distributions, no reweighting of second-order sequences was justified in view of the small number of observations in some of the sequence conditions.

\section{DISCUSSION}

The purpose of the present study was to examine whether FP-RT functions observed under different FP distributions can be predicted one from another by reweighting sequential effects. If so, the differences between observed FP-RT functions would mainly reflect differential unintentional influences of trace conditioning. To the extent the reweighting procedure fails to bridge the difference between the FP-RT functions, a role of another process, such as temporal orienting, is indicated in addition to, or perhaps overruling, trace conditioning. In what follows, we first comment on the sequential effects and FP-RT functions observed within FP distributions. Then we discuss the results of our reweighting procedure.

\section{Sequential Effects Within Distributions of FP}

The observations of the present study were generally consistent with earlier findings. In particular, we replicated the basic findings of sequential effects (e.g., Baumeister \& Joubert, 1969; Karlin, 1959; Los et al., 2001; Los \& Van den Heuvel, 2001; Van der Lubbe et al., 2004; Woodrow, 1914; Zahn et al., 1963). As Figure 1 shows, responding was slower when $\mathrm{FP}_{n-1}$ was longer than $\mathrm{FP}_{n}$ relative to when $\mathrm{FP}_{n-1}$ was equally long or shorter than $\mathrm{FP}_{n}$. This effect was particularly strong for the shortest $\mathrm{FP}_{n}$. For the $\mathrm{FP}_{n}$ of $600 \mathrm{msec}$, it was much weaker but in the expected direction. That is, RT was slightly longer when trial $n-1$ was either a catch trial or a trial with an FP of $1,200 \mathrm{msec}$ than when $\mathrm{FP}_{n-1}$ was either 300 or $600 \mathrm{msec}$. For the $\mathrm{FP}_{n}$ of 1,200 msec, the expected sequential effect was also observed when it is assumed that a catch trial constitutes a long FP that extends beyond 1,200 msec. Thus, RT for the FP of 1,200 msec was relatively long when a catch trial occurred on the preceding trial - that is, when the imperative moment was bypassed during $\mathrm{FP}_{n-1}$ (see Correa, Lupiáñez, Milliken, \& Tudela, 2004, and Los, 2004, for a similar result).

Two findings appear to slightly deviate from what is usually found. First, responding at the longest FP was relatively slow. The usual finding is that RT decreases monotonically with FP under the uniform distribution, while

Table 2

The RT Difference Before and After Reweighting the FP-RT Functions, Along With the Percentage Gain That is Obtained by Reweighting

\begin{tabular}{lccc}
\hline \multicolumn{1}{c}{ Prediction } & Total RT Difference & After Reweighting & Percentage Gain \\
\hline Uniform predicts peaked & 24.10 & 24.20 & -0.42 \\
Uniform predicts exponential & 28.11 & 23.31 & 17.09 \\
Exponential predicts uniform & 28.11 & 27.32 & 2.79 \\
Exponential predicts peaked & 35.45 & 35.68 & -0.65 \\
Peaked predicts uniform & 24.10 & 20.13 & 16.48 \\
Peaked predicts exponential & 35.45 & 31.82 & 10.23 \\
\hline
\end{tabular}


being independent of FP under the exponential distribution (e.g., Baumeister \& Joubert, 1969; Näätänen, 1971; Trillenberg et al., 2000). Second, RT was longer when the $\mathrm{FP}_{n}$ of 1,200 msec was preceded by a shorter $\mathrm{FP}_{n-1}$ than when preceded by an equally long $\mathrm{FP}_{n-1}$. The more common finding is an absence of sequential effects for the longest FP (e.g., Los et al., 2001; Van der Lubbe et al., 2004; Zahn et al., 1963).

One might argue that these deviant findings stem from the fact that we used a geometric progression of FPs (i.e., $300,600,1,200 \mathrm{msec}$ ) instead of the more common arithmetic progression. As a result, the latest critical moment was more isolated from the earliest two critical moments than is usually the case, which might imply that the preparatory state at the end of a long FP cannot profit from possible residual preparatory activation generated at the earlier critical moments. However, we were able to rule out this possibility in a pilot study, in which we observed the same deviations under an arithmetic progression of FPs (i.e., 300, 600, and $900 \mathrm{msec}$ ).

A more likely reason for the deviant findings is the rather high proportion of catch trials in our design, which varied from $11 \%$ in the peaked distribution to $25 \%$ in the uniform distribution. In fact, Correa et al. (2004) used the variable-foreperiod design with FPs of 350,850 , or $1,350 \mathrm{msec}$ and observed the typical downward sloping FP-RT function in a condition without catch trials and an upward sloping FP-RT function in a condition with $25 \%$ catch trials. The sequential effects in the condition with catch trials were very similar to those of the present study (as well as to those observed by Los, 2004). However, Trillenberg et al. (2000) used precisely the same distributions as in the present study, but still observed the more common FP-RT functions without RT delay for the longest FP. ${ }^{3}$ Thus, it remains to be established in future studies what design feature may account for the discrepancy between Correa et al.'s and the present findings on the one hand and Trillenberg et al.'s findings on the other hand.

While the upward slope of the final part of the FP-RT function may be less typical, it is not inconsistent with the trace conditioning model outlined in the Introduction. According to this model, bypassing a critical moment during FP entails extinction of the conditioned strength associated with that moment. Consistent with this principle is our observation of a particularly long RT for the FP of $1,200 \mathrm{msec}$ when the preceding trial was a catch trial (for similar results, see Correa et al., 2004; Los, 2004). Furthermore, our analysis of higher order sequential effects in the uniform condition revealed that RT for the $\mathrm{FP}_{n}$ of 1,200 msec increased when preceded by an increasing number of catch trials and decreased when preceded by an increasing number of trials with an FP of 1,200 msec. These findings support the view that the conditioned strength associated with the latest critical moment is subject to strong extinction as that moment is bypassed during FP on a catch trial. Recovery from the resulting low conditioned strength awaits reinforcement, which is contingent upon using the latest critical moment for the presentation of $\mathrm{S}_{2}$.
The magnitude of these (higher order) sequential effects suggests that trace conditioning dynamics may go a long way to explain Correa et al.'s (2004) finding of a downward-sloping FP-RT function in a condition without catch trials and an upward-sloping FP-RT function in a condition with catch trials. Whether the difference between these functions can be bridged completely by higher order sequential effects remains to be established. One indication that this might be the case comes from a study by Alegria (1978), who varied the percentage of catch trials (being $0 \%, 22 \%$, or $77 \%$ ) in a choice reaction task with a single FP of $700 \mathrm{msec}$. Alegria observed that the difference in mean RT between these conditions could be attributed completely to (higher order) sequential effects.

The only finding that challenges the conditioning account is that responding at the earliest critical moment is faster after a catch trial than after an FP of $1,200 \mathrm{msec}$ (see Figure 1, left panel; see Correa et al., 2004, and Los, 2004, for similar findings). Given that we conceived of a catch trial as a trial with an FP that exceeds $1,200 \mathrm{msec}$, one would expect responding at the earliest critical moment to be slower, if anything, after a catch trial than after a trial with an FP of 1,200 msec. Our finding therefore suggests that the execution of the response contributes to the extinction of the conditioned strength associated with a bypassed critical moment - a feature that is not part of the conditioning account in its present form.

\section{Reweighting Sequential Effects Across Distributions of FP}

The major question of the present study was to what extent FP-RT functions from two different FP distributions can be aligned after equating the relative contribution of specific intertrial transitions. As it turned out, reweighting sequential effects, according to Equation 1, accounted at best for only a modest percentage of the total difference between two FP-RT functions. In fact, the maximum percentage of gain we obtained by this procedure was $17.09 \%$, when the reweighted uniform FP-RT function predicted the exponential FP-RT function. Even though second-order reweighting (according to the equation given in note 2) enhanced this value by about $10 \%$, it is clear that it rendered the largest part of the difference unexplained. Furthermore, given that reweighting yielded even less successful predictions for other pairs of distributions, it is clear that the difference between FP-RT functions under different distributions cannot be bridged by reweighting alone.

This conclusion illustrates the merits of the reweighting procedure. Although it is possible in principle to draw the same conclusion from inspection of the sequential effects presented in Figure 1, it is not transparent from this figure that sequential effects have such a small share in the distribution effect. In fact, this lack of transparency may explain why previous studies have arrived at conflicting answers to the question of whether distribution effects have a status different from sequential effects. Whereas Drazin (1961) and Baumeister and Joubert (1969) judged 
the difference between the FP-RT functions under their different distributions to be bridgeable by reweighting sequential effects, Zahn and Rosenthal (1966) judged this difference to be unbridgeable. The present application of the reweighting procedure unambiguously settles the issue in favor of the latter viewpoint. That is, to fully account for the difference between the FP-RT functions observed under the different FP distributions, more than one process should be postulated.

What is the nature of these processes? As argued before, a simple trace conditioning model can effectively account for sequential effects (see also Los et al., 2001), and recent studies have supported some of its basic assumptions (Los, 2005; Los \& Heslenfeld, 2005; Los \& Van den Heuvel, 2001). These studies have qualified the sequential effect as an unintentional consequence of an extinction process that operates during FP on the conditioned strength associated with bypassed critical moments. However, the contribution of trace conditioning to the distribution effect is a minor one, given that sequential effects make up for only a small part of the distribution effect. The obvious candidate for explaining the remaining part of the distribution effect is temporal orienting (e.g., Nobre, 2001). In particular, it seems clear that different distributions induce participants to allocate resources to different points in time.

The only reservation we have in calling this process temporal orienting concerns the role of intentionality. On the one hand, it could be that the allocation of resources reflects an intentional process, driven by a subjective expectancy about the most likely duration of the impending FP (e.g., Correa et al., 2004; Milliken et al., 2003; Näätänen \& Merisalo, 1977; Zahn \& Rosenthal, 1966). In this view, participants use their knowledge of the FP distribution to allocate resources to the critical moment with the highest a priori probability. On the other hand, it could be that the process of allocating resources to different points in time is an unintentional process, independent of the participant's subjective expectancy. In this view, behavior is unintentionally influenced by both the local (sequential effect) and the global (distribution effect) history of preceding FPs. Since the present data do not permit us to distinguish between these two possibilities, we will use in what follows the term temporal orienting without recourse to its surmised intentional nature.

The present findings provide several clues about the achievements of temporal orienting. A close inspection of Figure 2 makes clear that the difference between each pair of FP-RT functions was largest at those critical moments where temporal orienting is supposed to operate under the exponential and peaked distributions. In particular, the difference between the uniform and exponential distributions was particularly large at the early critical moment. This difference was slightly reduced after reweighting sequential effects, but by far the largest part of the initial difference persisted. We attribute this difference to temporal orienting at the earliest critical moment under the exponential distribution. Similarly, the difference in mean RT for the second critical moment between the peaked and uniform distributions could also not be attributed to sequential effects. This again suggests a dominant role for temporal orienting at the central critical moment under the peaked distribution.

The difference in RT between distributions was much smaller at those critical moments where temporal orienting is supposed to be absent. This applies to the latest critical moment for all distributions, the second critical moment when comparing the uniform and exponential distributions, and the first critical moment when comparing the peaked and uniform distributions. Furthermore, to the extent these differences are reliable at all, there is some indication that they might be bridged by reweighting sequential effects. This is particularly visible for the peaked distribution in predicting the FP-RT function under the uniform distribution (Figure 2, middle panel). However, this reduction was not as impressive for the other comparisons, which might reflect a modest contribution of temporal orienting to the state of preparation even at these critical moments.

The data also provide some clues about the way temporal orienting interacts with the trace conditioning process at those critical moments that are most frequently used under the exponential and peaked distributions. With respect to the exponential distribution, the data suggest that temporal orienting attenuates sequential effects, similar to the effect of a valid cue under a uniform distribution (Los \& Heslenfeld, 2005; Los \& Van den Heuvel, 2001). As shown by the left panel of Figure 1, the difference between distributions was especially marked when $\mathrm{FP}_{n-1}$ was longer than $\mathrm{FP}_{n}$. Apparently, under the exponential distribution, the participants oriented the earliest critical moment also after a trial with a longer FP, thus attenuating sequential effects. In turn, this explains why reweighting sequential effects failed to bridge the difference at the earliest critical moment between the exponential distribution on the one hand and the uniform and peaked distributions on the other. However, the data argue against a similar role for temporal orienting under the peaked distribution, where, if anything, sequential effects for the second critical moment turned out to be larger than under the other distributions (Figure 1, middle panel). The main reason why reweighting failed in this case seems to be that $\mathrm{FP}_{n-1}$ did not contribute very much to RT for the middle FP in the first place.

In conclusion, sequential effects, which are supposed to reflect processes of trace conditioning, were present under each distribution but accounted for only a small part of the difference between distributions. Therefore, the data indicate a dominant role of temporal orienting in accounting for the effect of FP distribution. The findings further suggest that temporal orienting may have an independent positive effect on performance in addition to attenuating the negative consequences of intertrial transitions, as earlier observed in cuing studies. 
Finally, we want to point out that the method we developed may have relevance for fields of research other than nonspecific preparation. For instance, within the field of visual attention, it is a well-known phenomenon that irrelevant singletons have a distracting effect on search for a target (e.g., Bacon \& Egeth, 1994; Folk, Remington, \& Johnston, 1992; Theeuwes, 1991, 1992). This effect is strongly reduced when the identity of the target is held constant within a block of trials (pure blocks), relative to when it is varied (mixed blocks). On the basis of these findings, adherents of a top-down view of visual attention argued that preknowledge of the target identity can be used to overcome unintentional distractor effects (e.g., Bacon $\&$ Egeth, 1994). By contrast, adherents of a bottom-up view (e.g., Theeuwes, 1992) argued that intertrial priming can fully account for the larger distractor effects in mixed blocks. The reweighting procedure may help to shed some light on this controversy. Analogous to the present study, the frequency of specific intertrial transitions can be varied, after which the relative contributions of bottom-up and top-down processes could be determined by reweighting sequential effects.

\section{REFERENCES}

AlEgRIA, J. (1975). Sequential effects of foreperiod duration: Some strategical factors in tasks involving time uncertainty. In P. M. A. Rabbitt \& S. Dornic (Eds.), Attention and performance V (pp. 1-10). London: Academic Press.

Alegria, J. (1978). Sequential effects of catch-trials on choice reaction time. Acta Psychologica, 42, 1-6.

BACON, W. F., \& Egeth, H. E. (1994). Overriding stimulus-driven attentional capture. Perception \& Psychophysics, 55, 485-496.

BAUMEISTER, A., \& JouberT, C. (1969). Interactive effects on reaction time of preparatory interval length and preparatory interval frequency. Journal of Experimental Psychology, 82, 393-395.

Beringer, J. (1992). Entwurf einer Anwendersprache zur Steuerung psychologischer Reaktionszeitexperimente [Design of an application language for conducting psychological reaction time experiments]. Frankfurt am Main: Lang.

Correa, Á., Lupiáñez, J., Milliken, B., \& Tudela, P. (2004). Endogenous temporal orienting of attention in detection and discrimination tasks. Perception \& Psychophysics, 66, 264-278.

Coull, J. T., Frith, C. D., Büchel, C., \& Nobre, A. C. (2000). Orienting attention in time: Behavioural and neuroanatomical distinction between exogenous and endogenous shifts. Neuropsychologia, 38, 808-819.

Coull, J. T., \& Nobre, A. C. (1998). Where and when to pay attention: The neural systems for directing attention to spatial locations and to time intervals as revealed by both PET and fMRI. Journal of Neuroscience, 18, 7426-7435.

DrazIN, D. (1961). Effects of foreperiod, foreperiod variability and probability of stimulus occurrence on simple reaction time. Journal of Experimental Psychology, 62, 43-50.

Elithorn, A., \& LaWrence, C. (1955). Central inhibition-Some refractory observations. Quarterly Journal of Experimental Psychology, 7, 116-127.

Folk, C., Remington, R. W., \& Johnston, J. C. (1992). Involuntary covert orienting is contingent on attentional control settings. Journal of Experimental Psychology: Human Perception \& Performance, 18, 1030-1044.

GotTSDANKER, R. (1975). The attaining and maintaining of preparation. In P. M. A. Rabbitt \& S. Dornic (Eds.), Attention and performance $V$ (pp. 33-49). London: Academic Press.

JuRKOWSKI, A. J., STEPP, E., \& HACKLEY, S. A. (2005). Variable forepe- riod deficits in Parkinson's disease: Dissociation across reflexive and voluntary behaviors. Brain \& Cognition, 58, 49-61.

KaRLIN, L. (1959). Reaction time as a function of foreperiod duration and variability. Journal of Experimental Psychology, 58, 185-191.

KingStOne, A. (1992). Combining expectancies. Quarterly Journal of Experimental Psychology, 44A, 69-104.

Los, S. A. (1996). On the origin of mixing costs: Exploring information processing in pure and mixed blocks of trials. Acta Psychologica, 94, $145-188$.

Los, S. A. (2004). Inhibition of return and nonspecific preparation: Separable inhibitory control mechanisms in space and time. Perception \& Psychophysics, 66, 119-130.

Los, S. A. (2005). Bypassing the critical moment: Response inhibition accounts for sequential effects in nonspecific preparation. Manuscript submitted for publication.

Los, S. A., \& Heslenfeld, D. J. (2005). Intentional and unintentional contributions to nonspecific preparation: Electrophysiological evidence. Journal of Experimental Psychology: General, 134, 52-72.

Los, S. A., KNoL, D. L., \& Boers, R. M. (2001). The foreperiod effect revisited: Conditioning as a basis for nonspecific preparation. Acta Psychologica, 106, 121-145.

Los, S. A., \& VAN Den Heuvel, C. E. (2001). Intentional and unintentional contributions to nonspecific preparation during reaction time foreperiods. Journal of Experimental Psychology: Human Perception \& Performance, 27, 370-386.

LucE, R. D. (1986). Response times. New York: Oxford University Press.

Machado, A. (1997). Learning the temporal dynamics of behavior. Psychological Review, 104, 241-265.

Milliken, B., Lupiáñez, J., Roberts, M., \& Stevanovski, B. (2003). Orienting in space and time: Joint contributions to exogenous spatial cuing effects. Psychonomic Bulletin \& Review, 10, 877-883.

Miniussi, C., Wilding, E. L., Coull, J. T., \& Nobre, A. C. (1999). Orienting attention in time: Modulation of brain potentials. Brain, 122, 1507-1518.

NäÄTÄNEN, R. (1970). The diminishing time-uncertainty with the lapse of time after the warning signal in reaction-time experiments with varying fore-periods. Acta Psychologica, 34, 399-419.

NÄÄTÄNEN, R. (1971). Nonaging foreperiod and simple reaction time. Acta Psychologica, 35, 316-327.

NÄÄTÄNEN, R., \& MERISALO, A. (1977). Expectancy and preparation in simple reaction time. In S. Dornic (Ed.), Attention and performance $V I$ (pp. 115-138). Hillsdale, NJ: Erlbaum.

NiEMI, P., \& NÄÄTÄNEN, R. (1981). Foreperiod and simple reaction time. Psychological Bulletin, 89, 133-162.

Nobre, A. C. (2001). Orienting attention to instants in time. Neuropsychologia, 39, 1317-1328.

Sperling, G., \& Dosher, B. A. (1986). Strategy and optimization in human information processing. In K. R. Boff, L. Kaufman, \& J. P. Thomas (Eds.), Handbook of perception and human performance: I. Sensory processes and perception (pp. 2.1-2.65). New York: Wiley.

STERnBerG, S. (2001). Separate modifiability, mental modules, and the use of pure and composite measures to reveal them. Acta Psychologica, 106, 147-246.

Stevens, J. (1992). Applied multivariate statistics for the social sciences. Hillsdale, NJ: Erlbaum.

STILITZ, I. (1972). Conditional probability and components of RT in the variable foreperiod experiment. Quarterly Journal of Experimental Psychology, 24, 159-168.

Theeuwes, J. (1991). Cross-dimensional perceptual selectivity. Perception \& Psychophysics, 50, 184-193.

Theeuwes, J. (1992). Perceptual selectivity for color and form. Perception \& Psychophysics, 51, 599-606.

Trillenberg, P., Verleger, R., Wascher, E., Wauschkuhn, B., \& Wessel, K. (2000). CNV and temporal uncertainty with "ageing" and "nonageing" S1-S2 intervals. Clinical Neurophysiology, 111, 12161226.

VAN der Lubbe, R. H. J., Los, S. A., JaśKowski, P., \& Verleger, R. (2004). Being prepared on time: On the importance of the previous foreperiod to current preparation, as reflected in speed, force, and 
preparation-related brain potentials. Acta Psychologica, 116, $245-$ 262.

Woodrow, H. (1914). The measurement of attention. Psychological Monographs, 17(5, Whole No. 76), 1-158.

ZAHN, T. P. (1970). Effects of reductions in uncertainty on reaction time in schizophrenic and normal subjects. Journal of Experimental Research in Personality, 4, 135-143.

Zahn, T. P., \& RosenthaL, D. (1966). Simple reaction time as a function of the relative frequency of the preparatory interval. Journal of Experimental Psychology, 72, 15-19.

ZahN, T. P., RosenthaL, D., \& SHaKow, D. (1963). Effects of irregular preparatory intervals on reaction time in schizophrenia. Journal of Abnormal \& Social Psychology, 67, 44-52.

\section{NOTES}

1. The trace conditioning account we present here can be conceived of as a simplified version of Machado's (1997) formal model. In particular, according to the formal model, the influence of extinction and reinforcement on the state of conditioning is gradual over the time scale rather than restricted to fixed points in time. This allows the formal model to account for the precise shape of FP-RT function observed when FP is varied both within and between blocks of trials (see Los et al., 2001, for details).

2. The calculation of the predicted RT after reweighting sequential effects up until the second order is a straightforward extension of Equation 1 and is given by

$$
\hat{\sim m}(f \leftarrow g)=\sum_{j} \sum_{k} \frac{\mathrm{RT}_{i j k}(g) \times N_{i j k}(f)}{N_{i}(f)},
$$

where $k$ refers to the FP on trial $n-2$.

3. Unfortunately, Trillenberg et al. (2000) did not report sequential effects. Instead, they went on to show that RT decreases linearly with an increasing conditional probability of $\mathrm{S}_{2}$ occurrence (see also Stilitz, 1972). However, as long as the contribution of sequential effects is not taken into account, this finding does not warrant the inference that the conditional probability also determines the state of nonspecific preparation. As can be easily verified, the conditional probability of $\mathrm{S}_{2}$ occurrence is highly correlated with the probability that $\mathrm{FP}_{n}$ is longer than or equal to $\mathrm{FP}_{n-1}(.83$ in the present case and that of Trillenberg et al., 2000).

(Manuscript received June 25, 2004; revision accepted for publication February 3, 2005.) 